\title{
Enhanced Rieger type periodicities' detection in X-ray solar flares and statistical validation of Rossby waves' existence
}

\author{
Michaila Dimitropoulou ${ }^{1}$, Xenophon Moussas ${ }^{2}$ and Dafni Strintzi ${ }^{3}$ \\ ${ }^{1}$ University of Athens, Department of Physics, GR-15483, Athens, Greece, \\ email: michaila.dimitropoulou@nsn.com \\ ${ }^{2}$ University of Athens, Department of Physics, GR-15483, Athens, Greece, \\ email: xmoussas@phys.uoa.gr \\ ${ }^{3}$ National Technical University of Athens, GR-15773, Athens, Greece, \\ email: dafni_strintzi@yahoo.com
}

\begin{abstract}
The known Rieger Periodicity (ranging in literature from 150 up to 160 days) is obvious in numerous solar indices. Many sub-harmonic periodicities have also been observed $(128-, 102-, 78-$, and $51-$ days) in flare, sunspot, radio bursts, neutrino flux and flow data, coined as Rieger Type Periodicities (RTPs). Several attempts are focused to the discovery of their source, as well as the explanation of some intrinsic attributes that they present, such as their connection to extremely active flares, their temporal intermittency as well as their tendency to occur near solar maxima. In this paper, we link the X-ray flare observations made on Geosynchronous Operational Environmental Satellites (GOES) to an existing theoretical model (Lou 2000), suggesting that the mechanism behind the Rieger Type Periodicities is the Rossby Type Waves. The enhanced data analysis methods used in this article (Scargle-Lomb periodogram and Weighted Wavelet Z-Transform) provide the proper resolution needed to argue that RTPs are present also in less energetic flares, contrary to what has been inferred from observations so far.
\end{abstract}

Keywords. Sun flares, Rieger-Type periodicities, Rossby-type waves

\section{Introduction}

In 1984 Rieger (Rieger et al. 1984) was the first to reveal a 158-day periodicity in the sun, while studying $\gamma$-ray flare data from Solar Maximum Mission (SMM) in solar cycle 21 (C21). Approximately the same periodicity was also discovered in X-ray flares data taken from the Geosynchronous Operational Environmental Satellites (GOES) for the same solar cycle (Rieger et al. 1984). In the context of these attempts, it is noticeable that apart from the Rieger Periodicity itself, numerous other "relative" periodicities were discovered, such as 128-, 102-, 78-, and 51-day periodicities (Bai \& Sturrock 1991,Bai 1992). The relevance of these periodicities to the classic Rieger one is that all of them are approximately integer multiples of a principle 25-day periodicity. This remarkable attribute led to coining these periodicities as "Rieger Type Periodicities" (RTPs). A series of additional conclusions came to the surface over the years of observational studies, the most important of which being that Rieger Periodicity was mainly seeked in highly explosive flares. Especially when it comes to X-ray flare data from GOES - which is going to be the data source for this study as well - the existence of Rieger periodicity was mainly investigated in flares of class $\geqslant M$ (Rieger et al. 1984, Landscheidt 1987 , Bai 2003). Consequently, Rieger periodicity was connected to highly energetic flares, which 
are presumably triggered by the emergence of photospheric magnetic flux with the same period (Ballester et al. 2002, Ballester et al. 1999).

Other than their existence, their intermittency and their tendency to occur near solar maxima, there is presently no solid ground for RTPs' theoretical explanation, although numerous models have been proposed over the years. Lou (Lou 2000) attempted to eliminate this distance from the recorded observations. His suggestion was that such periodicities are linked to large-scale equatorially trapped Rossby-type waves. For typical solar parameters, Lou's theoretical model results to periodicities which are very close to the observed ones. More specifically, if sheer Rossby waves are assumed, then the families of periods, which beget from the dispersion relation of these waves in the sun, are:

$$
P_{r} \cong P_{@}\left(\frac{|m|}{2}+\frac{(2 n+1) \Omega_{@} R_{@}}{|m|(g D)^{1 / 2}}\right),
$$

where $P_{@}=\frac{2 \pi}{\Omega_{@}} \sim 25.1$ days is the solar sidereal rotation period $\left(\Omega_{@}\right.$ being the solar rotation frequency), $n \geqslant 0$ is an integer indicating the number of the considered nodes, $m$ is an integer related to the wavenumber $k_{x}=\frac{m}{R_{@}}$ with $m \geqslant 1, R_{@}=7 \times 10^{10} \mathrm{~cm}$ is the solar radius, $g=2.7 \times 10^{4} \mathrm{~cm} / \mathrm{s}^{2}$ is the solar surface gravity acceleration and $D=500 \mathrm{~km}$ is the average thickness of the photosphere. It is noticeable that for $m=4,6,8,10,12$ the $P_{r}=53.4,77.4,102.000,126.780,151.667$ day-periods are produced very consistently by Lou's model in agreement with the observations (Bai \& Sturrock 1991, Bai 1992), whereas the odd-valued modes are not so frequently observed.
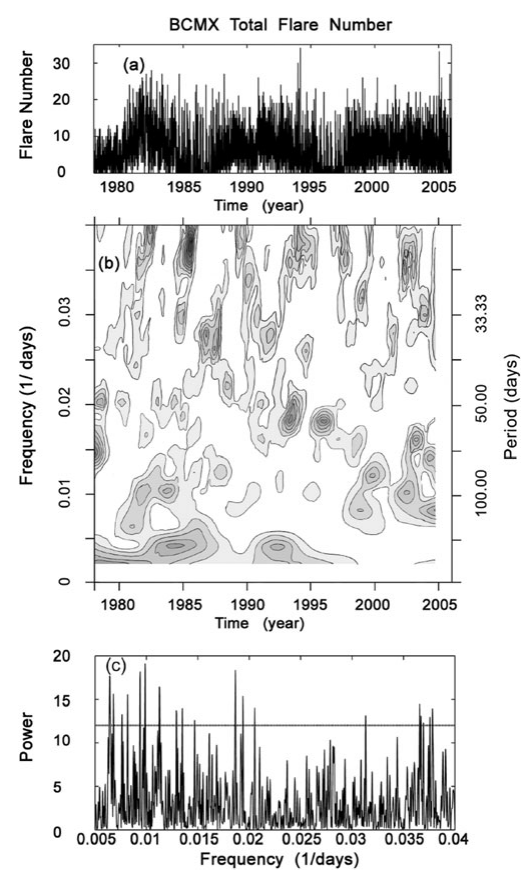

Figure 1. Plot of: (a) Time Series of B,C,M,X summed daily flare numbers, (b) WWZ Wavelet Analysis of B,C,M,X summed daily flare numbers and (c) Scargle-Lomb periodogram for the same data. The dash-dotted line marks the 0.005 level of significance. 


\section{Motivation and scope}

Yet, even Lous recent model leaves several questions unanswered. First and foremost, is it really so, that the RTPs are indeed related to already established active regions, leading mainly to extreme flares? Does the periodic emergence of magnetic flux indeed prefer already formed active regions, thus building complex magnetic configurations which are prompt to produce highly energetic flares? In other words, would it be also possible to clearly observe RTPs in flares of class M, or would RTPs be less prominent in this case? In addition, if we attribute RTPs to Rossby waves according to Lous model, then why observations favor waves with even $\mathrm{m}$ values, thus excluding other periodicities which are theoretically possible? The scope of this work is to provide answers to the abovementioned open questions.

\section{Data and methods}

In this work, we apply the ScargleLomb periodogram and we test for the first time Wavelet Weighted Z-Transform (WWZ) on X-ray flare data, derived from GOES since 1978. First, the sum of flares per day is considered. This sum can be calculated either over all classes of flares (B, C, M, X) or over the most energetic ones (M, X). Similar data analysis has been attempted several times in the past, like by Bai in 2003 (Bai 2003). This work only considers a prolonged time-period reaching 2006 and adopts daily instead of weekly flare event sums. The novelty presented in this work is the analysis of flares, taken as intensities with time-resolution of $1 \mathrm{~min}$ and not as sums of flare events per day. This is possible through the enhanced WWZ method, which is able to handle consistently events which are not regularly distributed in time. The reason for selecting such an analysis is that it can indeed classify flares in a physically continuous
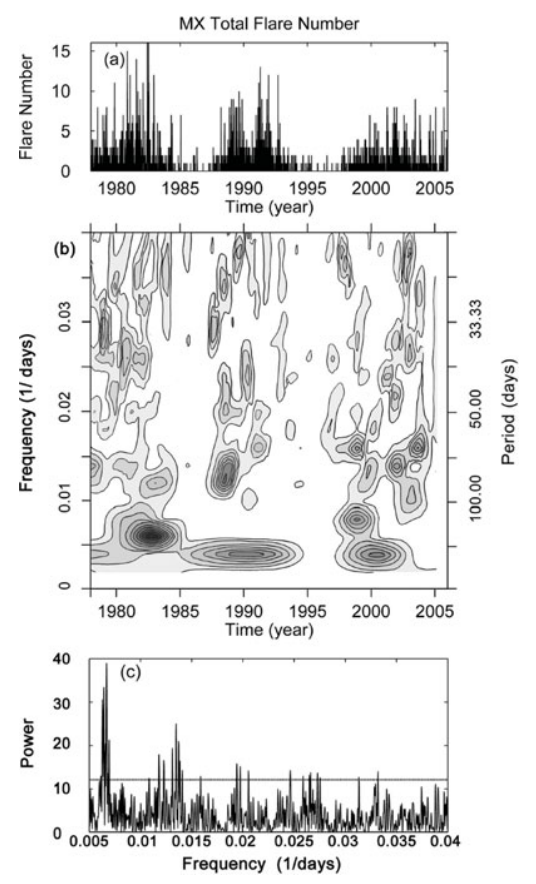

Figure 2. Plot of: (a) Time Series of M,X summed daily flare numbers, (b) WWZ Wavelet Analysis of M,X summed daily flare numbers and (c) Scargle-Lomb periodogram for the same data. The dash-dotted line marks the 0.005 level of significance. 
way, without manufacturing artificial sums. In fact, this kind of methodology is applied both for all classes of flares (B, C, M, X) and exclusively for the less energetic ones (B and $\mathrm{C}$ ), in order to investigate whether RTPs are prominent also in the latter case.

\section{Conclusions}

In this work, flare data have been analyzed with high resolution methods and were compared with up-to-date theoretical models, targeting RTPs. Starting from Lous theoretical model (Lou 2000), we investigated whether even m periodicities are indeed strangely favored in the Sun. We have proved that when extensive data series are used, odd $\mathrm{m}$ periodicities are also frequent and considerably significant. This conclusion places the theoretically foreseen odd $\mathrm{m}$ periodicities in the position of normal subharmonics of the 25-d period, exactly as even ones, also from the observational point of view.

As far as the analysis of the X-ray flare data is concerned, the concept of applying statistics on the intensity of the events instead of their summed daily number is tested using the powerful tool of WWZ for the first time, along with the well-established ScargleLomb periodogram. Such methods allow the exploitation of the minute resolution offered by GOES. This enhanced analysis shows that RTPs are present also for weak flare events, thus decoupling these periodicities from magnetic field complexity and extremely active regions. Yet, the occurrence of RTPs in less ener- getic flares needs further theoretical justification. RTPs are observable both/either in sunspot areas and/or in sunspot groups depending on the solar cycle examined (Massi 2007). The former observation is linked with increased magnetic complexity, therefore it would fit closely to RTPs connected with energetic flares (i.e.Mand X class).
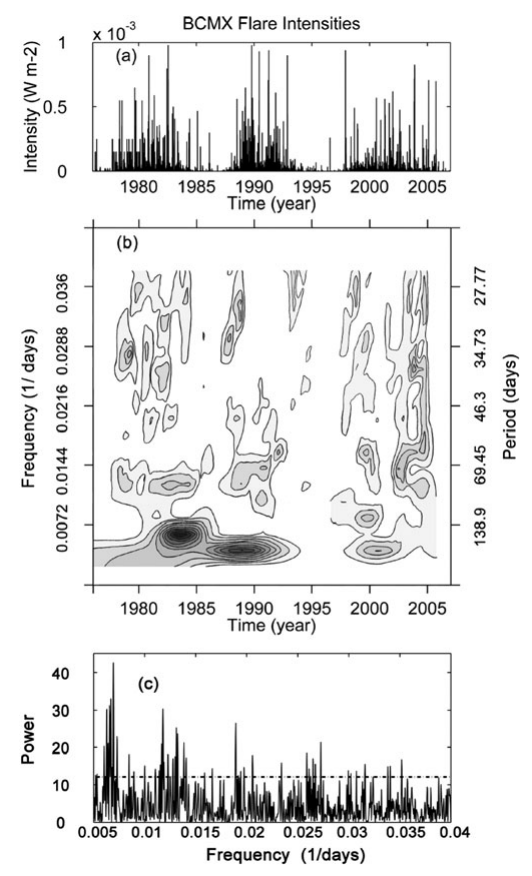

Figure 3. Plot of: (a) Time Series of B,C,M,X flare intensities (minute resolution), (b) WWZ Wavelet Analysis of B,C,M,X flare intensities (minute resolution) and (c) Scargle-Lomb periodogram for the same data. The dash-dotted line marks the 0.005 level of significance. 

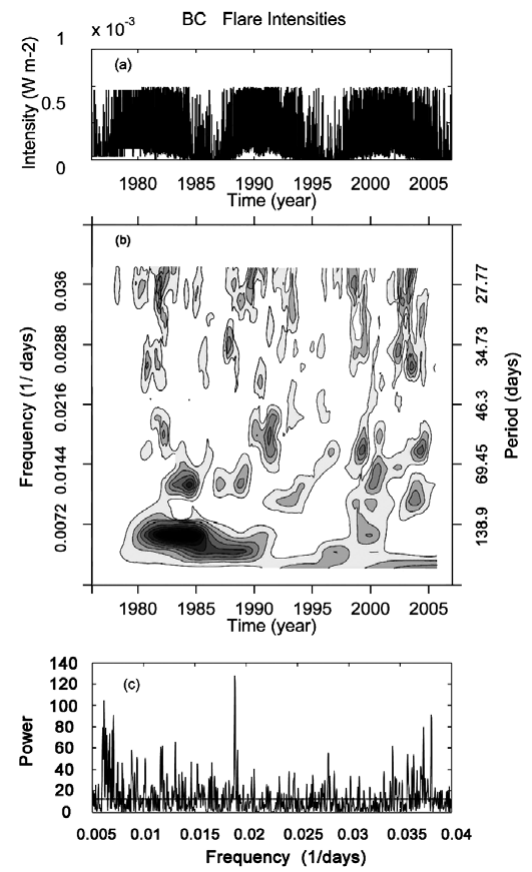

Figure 4. Plot of: (a) Time Series of B,C flare intensities (minute resolution), (b) WWZ Wavelet Analysis of B,C flare intensities (minute resolution) and (c) Scargle-Lomb periodogram for the same data. The dash-dotted line marks the 0.005 level of significance.

Even so, weak looploop interactions resulting from such highly complex magnetic configurations could indeed beget secondary less energetic flares (i.e. B and C class). The latter observation could fit to RTPs connected with any class of flares. The physical mechanism ambiguity of RTP occurrence in less energetic flares could be resolved with future simultaneous analysis on sunspot areas and groups.

The results begetting from Weighted Wavelet Z-Transforms also prove a consistent temporal localization of RTPs among the various analysed flare data series. The Rieger periodicities revealed in this work both in flare number as well as intensity analysis coincide very nicely in time. The temporal occurrence of Rieger periodicities is also consistently cross-checked with previous results (Rieger et al. 1984, Ballester et al. 1999, Ballester et al. 2002) coming from classic wavelet analysis on different solar indices. The prominence of Rieger periodicity in C21, C23 and its absence from C22 is also confirmed within the scope of this work.

\section{References}

Bai, T. 1992, ApJ, 388, L69

Bai, T. 2003, ApJ, 591, 406

Bai, T. \& Sturrock, P., 1991, Nature, 350, 141

Ballester, J. L., Oliver, R., \& Baudin, F. 1999, ApJ, 522, L153

Ballester, J. L., Oliver, R., \& Carbonell, M. 2002, ApJ, 566, 505

Landscheidt, T. 1987, Solar Phys., 107, 195

Lou, Y. Q. 2000, ApJ, 540, 1102

Massi, M. 2007, MemSAI, 78, 247

Rieger, E., Share, G. H., Forrest, D. J., Kanbach, G., Reppin, C., \& Chupp, E. L. 1984, Nature, 312,623 